\title{
Rare 2030 Final Policy Conference: Summary of the Recommendations of the Rare 2030 Foresight Study
}

\section{Evgenia Koutsouki}

Editor

Citation: EMJ. 2021;6[1]:12-14.

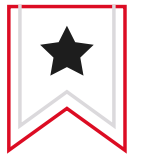

\begin{abstract}
MPROVING the lives of people living with a rare disease in Europe has been an overarching aim of the Rare 2030 Foresight Study, a two-year study that collected input from various partners, including patient groups, practitioners, and key opinion leaders. By identifying twelve political, economic, sociocultural, technological, ethical, and legal trends, the study produced eight recommendations to help establish new and improved policies for rare diseases in Europe.? The virtual Rare 2030 Final Policy Conference that took place on February $23^{\text {rd }}$ saw the various stakeholders come together to present and comment on these recommendations. ${ }^{2}$
\end{abstract}

\section{FRAMEWORK STRATEGY AND EARLY DIAGNOSIS}

The first of these recommendations pertains to long-term, integrated European and national plans and strategies for rare diseases. Kate Bushby, the lead researcher for the 2030 Project, explained that in light of new technology and progress in the field of rare diseases, there was a need to align these efforts into a new framework. It had become increasingly obvious that the previous framework and national plans were becoming outdated and inconsistent. Yann Le Cam, European Organisation for Rare Diseases (EURORDIS), emphasised that despite the progress made in the last 15 years, there are still unmet clinical needs for the 30 million people living with rare diseases in Europe. The new vision is to embed all the efforts in an integrated framework at the European level, which will then be supported nationally to ensure it is implemented on a day-to-day basis. New funding will ensure that there is a new cohesive plan for every person with a rare disease across Europe and will ensure that rare diseases remain a public health priority in Europe.

When it comes to the speed of and access to diagnosis, earlier, faster, and more accurate diagnosis is a key goal of the recommendations. An ambitious goal has been set to have every person with an identifiable rare disease diagnosed within six months of seeking medical attention. According to Le Cam, setting ambitious objectives will help to improve performance and potentially see the European Union (EU) become a world leader in biotechnology. Le Cam also highlighted the need to adopt a holistic approach to the needs and care of patients, powered by data: "As opposed to 10 years ago where process outcomes were measured, we are now in a position to have qualitative and quantitative objectives, and have a real impact 
on society, on quality of life, and on economies." Of course, diagnosis for people with currently undiagnosable conditions will remain an ongoing challenge; however, the recommendations state that despite the absence of a diagnosis, these people would still have access to the best possible care and support.

\section{EQUALITY OF ACCESS}

Access to services is a theme that runs throughout the recommendations, as the third recommendation addresses access to highquality healthcare. Adopting an approach to rare diseases at the EU level means that all citizens can have access to diagnosis, care, and treatment, whether close to home or across national borders. "It is a matter of equality, it is a matter of civil right. Every citizen with a disease has the right to be treated," commented Prof Maurizio Scarpa, MetabERN.

Putting people at the forefront of such an initiative is the key to its success; therefore, there is a great need to ensure equal opportunities and integration of people living with rare diseases, which is what the fourth recommendation focusses on. Giovanna Giuffrè, ISINNOVA, commented that trends observed during the coronavirus disease (COVID-19) pandemic can help for understanding challenges and opportunities that are relevant to patients with rare diseases. Giuffrè commented that during the pandemic there have been trends of increased inequality and reduced cohesion in healthcare, while at the same time opportunities have arisen in digital health, artificial intelligence, and multistakeholder collaboration in research.

\section{PARTNERSHIPS AND ADVOCACY}

The next recommendation focusses on establishing a culture in which people living with rare diseases can be empowered to actively participate in research and innovation, leading to the creation of partnerships in the public and private sectors. Le Cam commented that the fundamental change proposed by this recommendation would be a change from collaboration to partnering. "What we need for success is a change of culture for an active

\section{"It is a matter of equality, it is a matter of civil right. Every citizen with a disease has the right to be treated,"}




\section{"the Rare 2030}

Recommendations, with an

inclusive and collaborative

approach, can be a key

factor in actively spreading

awareness to debunk

stereotypes and stigmas." and meaningful participation of patients," Le Cam emphasised.

This need for partnership with patients and their representatives was reinforced by the findings of surveys that were carried out between 2016 and 2021, which have shown that 9 out of 10 patient representatives wish to be more involved in the research ecosystem, help researchers, and participate more in research projects as official partners and co-investigators.

Encouraging innovation and efficiency in the development of rare disease therapies would not only improve organisation, but would also help management of care. Creating greater incentives for research and promoting international partnerships between organisations and among different sectors are key to achieving this goal. Lucia Monaco, Fondazione Telethon, explained that emphasis should be placed on identifying unmet needs, "with particular attention to diseases that are neglected by research and development." Monaco also explained that "to accelerate excellent science in rare diseases, funders should support the translation of innovation from bench to the clinic and promote access to existing infrastructures."

\section{ACCESSIBLE DATA AND THERAPIES}

Over recent decades, the dissemination of data has become an important aspect of research and innovation. Data are the focus of the seventh recommendation: more specifically, the collection and integration of data on rare diseases. One of the key objectives is ensuring that such data are encoded under the uniform nomenclature of Orphanet. Integrating these data under a federated European system will encourage collaboration and help to promote progress in research and potential treatments. However, Bushby emphasised that "we are custodians of these data, they don't belong to us. They belong to the patient, they belong to the community, and making sure that sharing is done in a meaningful way for the right reasons is probably the best that we can hope to achieve as time goes on."

The final recommendation seeks to ensure that preventative technologies and therapies are accessible to all people in Europe living with a rare disease. Ultimately, the long-term goal is to form an environment ripe for investment, to establish robust pharmaceutical and biotechnology manufacturing presences in Europe, combined with better patient access and health monitoring.

Among the stakeholders consulted for these recommendations were the 2030 Young Citizens. Fanni-Laura Mäntylä, representing Rare 2030 Young Citizens, commented that "the Rare 2030 Recommendations, with an inclusive and collaborative approach, can be a key factor in actively spreading awareness to debunk stereotypes and stigmas."

It is clear that a new era is beginning for rare diseases in Europe, which will see a more integrated, accessible, and cross-border approach, encouraging innovation, research, and development in the field, while keeping people at the heart of the approach, leaving no European citizen behind.

\section{References}

1. Rare 2030: Foresight in Rare Diseases Policy; EURORDIS-Rare Diseases Europe. Recommendations from the Rare 2030 Foresight Study. 2021. Available at: http://download2.eurordis.org/rare2030/Rare2030_ recommendations.pdf. Last accessed: 8 March 2021.

2. Rare 2030: Foresight in Rare Diseases Policy. Feb 23 Final Policy Conference. 2021. Available at: https://www. rare2030.eu/key-events/feb-2021-policy-conference/. Last accessed: 8 March 2021. 